\title{
Regulação emocional em ambientes educacionais: um mapeamento sistemático
}

\author{
Helena Macedo Reis ${ }^{1}$, Renato Jum Ono Kassa, Patricia Jaques ${ }^{2}$, Seiji Isotani ${ }^{1}$, Ramílio \\ Filho $^{3}$ \\ ${ }^{1}$ ICMC/USP, São Carlos - SP - Brasil \\ ${ }^{2}$ PPGCA/UNISINOS, São Leopoldo - RS - Brasil \\ ${ }^{3}$ FATEC/TQ, Taquaritinga - SP - Brasil \\ renatojum@gmail.com, pjaques@unisinos.br \\ $\{$ helenamcd, sisotani\}@icmc.usp.br
}

\begin{abstract}
This paper aims to perform a survey of the scientific work carried out in the last 18 years in the area of emotional regulation in the context of computers in education. For this, a systematic mapping was carried out in the main national and international digital libraries. Initially, 1428 articles were collected, of which 34 were selected for final analysis. As a result, the current state of the art in emotional regulation in educational settings was presented. It has also been found that despite most studies, using Gross's theory of emotional regulation for emotional adjustment of the student within the educational environment, a significant number still do not use some theories. Even so, a large part of the studies that use a theory for a regulation did not reveal in what way applied as an emotional strategy. In addition, it was observed that most studies do not consider the context (e.g. algebra teaching) for regulation.
\end{abstract}

Resumo. Este artigo tem como objetivo realizar um levantamento dos trabalhos científicos, realizados nos últimos 18 anos, na área de regulação emocional no contexto de informática na educação. Para isso, um mapeamento sistemático foi conduzido nas principais bibliotecas digitais nacionais e internacionais. Inicialmente, foram coletados 1428 artigos, dos quais 34 foram selecionados para análise final. Como resultado, foi apresentado o atual estado da arte em regulação emocional nos ambientes educacionais. Também verificou-se que apesar da maioria dos estudos utilizarem a teoria de regulação emocional de Gross para a regulação emocional do aluno dentro do ambiente educacional, um número significativo ainda não usa quaisquer teorias. Mesmo assim, uma grande parte desses estudos que utilizam uma teoria para a regulação, não revelaram de qual maneira aplicavam as estratégias emocionais. Além disso, foi observado que a maior parte dos estudos não consideram o contexto (e.g. ensino de álgebra) para a regulação.

\section{Introdução}

As emoções desempenham um papel fundamental nos processos cognitivos, e se revelam essenciais durante a aprendizagem [Pessoa 2008]. Elas influenciam os estados psicológicos e biológicos, podendo aumentar a atenção dos alunos, bem como melhorar a memória 
VII Congresso Brasileiro de Informática na Educação (CBIE 2018)

Anais do XXIX Simpósio Brasileiro de Informática na Educação (SBIE 2018)

e o raciocínio [Oxford and Bolaños-Sánchez 2016]. Como consequência, as relações entre objetos ou ideias são feitas com mais facilidade, promovendo a eficiência e o rigor tanto na tomada de decisões, como na resolução de problemas [Isen 2000].

Entretanto, algumas emoções podem impactar negativamente no aprendizado. Quando o estudante experimenta emoções negativas (medo do fracasso, medo, tédio, pressões psicológicas de diversas naturezas, sensação de incompetência, tristeza, raiva, desespero, ansiedade, desgosto, etc.) sua performance de aprendizagem é reduzida [Gross and Thompson 2007]. Contudo, essas emoções são inevitáveis durante o processo de aprendizagem e acompanham o aluno em toda a sua vida acadêmica.

Para evitar que o aluno sinta emoções negativas e vivencie mais emoções positivas durante o aprendizado, diversas pesquisas têm focado na regulação emocional durante o ensino com o suporte computacional [Arguedas et al. 2016]. A regulação emocional pode ser definida como um processo pelo qual uma pessoa modifica a experiência de expressão emocional para promover bem estar pessoal. A regulação, aliada com o suporte computacional, pode produzir emoções mais otimistas, além de um melhor ganho de aprendizado [Malekzadeh et al. 2015].

Com o intuito de apresentar uma visão geral sobre as pesquisas que investigam a regulação emocional em ambientes educacionais computacionais, foi realizado um mapeamento sistemático ${ }^{1}$ das pesquisas existentes nos veículos brasileiros oficiais de divulgação científica na área (CBIE, RBIE e Renote) e internacionais (IEEExplore, ACM Digital Library, Scopus, Elsevier (via Science Direct) e Springer Link (via ScienceDirect)).

O objetivo deste artigo é analisar as pesquisas na área da regulação emocional em ambientes educacionais computacionais, identificando quais teorias e estratégias de regulação emocional vêm sendo utilizadas, como são usadas dentro desses sistemas, qual a forma de interação e quais os tipos de ambientes computacionais empregados. Além disso, espera-se identificar como as emoções são detectadas e em quais disciplinas a regulagem emocional tem sido empregada.

\section{O Processo do Mapeamento Sistemático}

Este estudo baseou-se nas diretrizes propostas por [Petersen et al. 2008] e foi conduzido de acordo com os cinco passos a seguir: (i) definição de questões de pesquisa, (ii) realização da pesquisa de estudos primários relevantes, (iii) triagem dos documentos, (iv) keywording dos resumos, e (v) a extração de dados e mapeamento.

Considerando que as questões de pesquisa devem exemplificar os objetivos do estudo de mapeamento, as seguintes questões foram elaboradas:

$\mathbf{Q P}_{1}$ : Quais teorias para regulagem emocional foram utilizadas?

$\mathbf{Q P}_{2}$ : Dentre estas teorias, quais estratégias de regulagem emocional foram utilizadas?

$\mathbf{Q P}_{3}$ : De que forma estas estratégias são utilizadas na regulação?

\footnotetext{
${ }^{1} \mathrm{O}$ mapeamento sistemático é uma metodologia que envolve a busca por literatura a fim de verificar a natureza, a extensão e a quantidade de estudos publicados (chamados de estudos primários) [Petersen et al. 2008].
} 
VII Congresso Brasileiro de Informática na Educação (CBIE 2018)

Anais do XXIX Simpósio Brasileiro de Informática na Educação (SBIE 2018)

$\mathbf{Q P}_{4}$ : Quais as principais formas de interação para a regulação emocional nos ambientes educacionais?

QP 5 : Quais os tipos de ambientes computacionais empregados?

$\mathbf{Q P}_{\mathbf{6}}$ : Como as emoções são detectadas?

$\mathbf{Q P}_{7}$ : Para quais disciplinas (domínios de conhecimento) a regulagem emocional tem sido empregada?

O processo de seleção dos artigos contou com três etapas de filtro. Essas etapas foram definidas para garantir que somente estudos sobre regulação emocional, aplicados à informática na educação, fossem contemplados para o mapeamento sistemático proposto neste artigo. As três etapas foram descritas como (i) busca por palavra chave, (ii) leitura dos artigos e (iii) verificação de equivalência entre artigos.

$\mathrm{Na}$ primeira etapa, foi realizada a busca por artigos que apresentavam em seus títulos, resumos e palavras-chave a string de pesquisa (("emotion regulation" OR "emotional regulation" OR "manage emotion" OR "adjust emotion" OR "adapt emotion") AND learning). A busca foi realizada nas seguintes bibliotecas digitais: IEEExplore, ACM Digital Library, Scopus, Elsevier (via Science Direct) e Springer Link (via Science Direct). Na língua portuguesa, a busca nesses mesmos campos foi realizada de forma manual por meio das palavras regula, adapt, afe, affe, ajus. As bibliotecas digitais utilizadas para se obter os estudos primários na língua portuguesa foram Simpósio Brasileiro de Informática na Educação (SBIE), Revista Novas Tecnologias na Educação (Renote) e Revista Brasileira de Informática na Educação (RBIE).

A pesquisa dos estudos primários foi conduzida durante os meses de fevereiro e março de 2018. Primeiramente foram retornados 1498 estudos primários das bibliotecas digitais. Dois autores deste estudo realizaram simultaneamente a leitura dos títulos, resumos e palavras-chave, de todos os artigos e cada um gerou a lista de estudos selecionados. Os dois autores discutiram sobre quais estudos deveriam ser selecionados a partir dos critérios de inclusão, e ao final o conjunto inicial de 1498 foi reduzido à somente 126 estudos realmente relacionados ao tópico investigado. Os critérios de inclusão utilizados, foram:

- Contribuições originais (se vários artigos relatam o mesmo estudo sem avaliações, modelos ou resultados diferentes, apenas o artigo mais recente é selecionado. Isso engloba artigos de um mesmo grupo de pesquisa de um único trabalho, se os artigos não apresentarem resultados ou contribuições diferentes);

- Artigos que envolvam estudo da regulação emocional na computação;

- Artigos que sejam do campo educacional;

- Estudos que estejam na língua portuguesa ou inglesa;

- Estudos primários e artigos científicos (relatórios técnicos, documentos na forma de resumos ou apresentações/slides e também estudos secundários, ou seja, revisões e mapeamentos sistemáticos da literatura, foram desconsiderados).

Os artigos não selecionados eram duplicados, estavam em outras línguas ou não envolviam informática no estudo. Além disso, apesar da string de pesquisa possuir a palavra learning, alguns artigos não eram sobre educação e apenas citavam estava palavra no texto, como um possível trabalho futuro. 
VII Congresso Brasileiro de Informática na Educação (CBIE 2018)

Anais do XXIX Simpósio Brasileiro de Informática na Educação (SBIE 2018)

Após esse passo, foi realizada novamente a leitura do resumo, introdução e conclusão dos 126 estudos selecionados, para assim, aplicar os critérios de inclusão.

A última etapa retornou como resultado $34 \operatorname{artigos}^{2}$, que foram selecionados para a realização desse mapeamento sistemático. A Figura 1 ilustra a quantidade de artigos publicados no tema, agrupados por ano de publicação. É possível observar que o assunto vem ganhando destaque e atenção da comunidade científica com o passar dos anos, tendo publicações anualmente a partir de 2011.

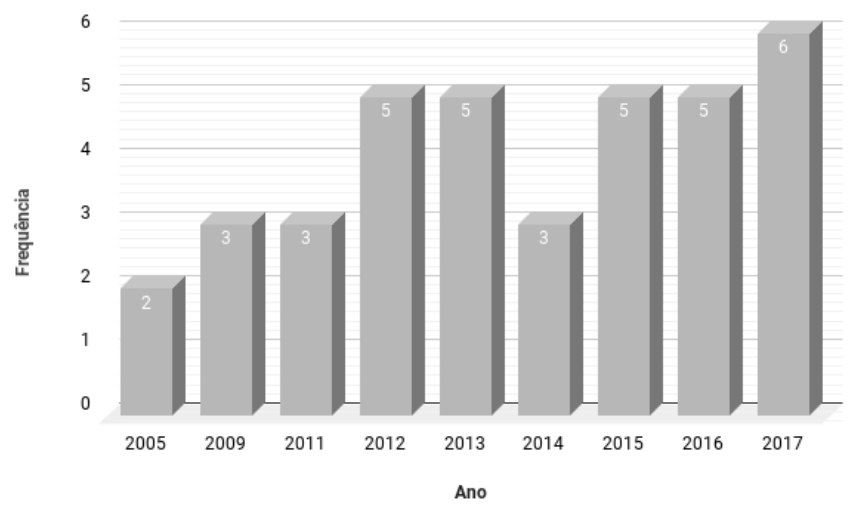

Figura 1. Seleção de estudos por ano e base

Os 34 artigos selecionados passaram por um novo processo de leitura para a extração de informações capazes de classificar os estudos em análise. Essa extração classificada tem como objetivo buscar os seguintes pontos: (i) apresentar as teorias para regulação emocional $\left(\mathbf{Q P} \mathbf{P}_{\mathbf{1}}\right)$, (ii) suas estratégias $\left(\mathbf{Q} \mathbf{P}_{\mathbf{2}}\right)$, (iii) como elas são utilizadas $\left(\mathbf{Q} \mathbf{P}_{\mathbf{3}}\right)$ e (iv) as formas de interação nos ambientes educacionais que regulam as emoções $\left(\mathbf{Q P}_{\mathbf{4}}\right)$; (v) classificar o tipo de ambiente $\left(\mathbf{Q P} \mathbf{P}_{5}\right)$, (vi) apresentar o método de detecção $\left(\mathbf{Q P} \mathbf{P}_{\mathbf{6}}\right)$ e (vii) apresentar as disciplinas em que a regulação emocional tem sido empregada $\left(\mathbf{Q} \mathbf{P}_{7}\right)$. Os resultados obtidos foram utilizados para embasar as respostas das questões de pesquisa deste mapeamento, descritas na Seção 3.

\section{Resultados e Discussões}

Nesta seção são descritos os resultados obtidos a partir da leitura e classificação dos 34 trabalhos selecionados. Para isso, esta seção está dividida em subseções, as quais cada uma descreve os resultados de acordo com uma questão de pesquisa.

\section{1. $\mathbf{Q P}_{1}$ : Teorias para regulação emocional considerados e $\mathbf{Q P}_{2}$ : Estratégias de regulação emocional}

Nessa etapa o objetivo era identificar quais são as teorias de regulação emocional que os sistemas educacionais vêm adotando (Tabela 1). Apesar da teoria de regulação emocional de Gross ser a mais utilizada, correspondendo a 16 estudos primários (43\%), é possível perceber que um número significativo (15 estudos primários, com 40\%) não se baseia em qualquer teoria psicológica sobre regulação emocional. O trabalho colaborativo com

\footnotetext{
${ }^{2}$ A lista completa de artigos pode ser visualizada em https : / / goo.gl / pt GHxW na aba "Selecionados".
} 
VII Congresso Brasileiro de Informática na Educação (CBIE 2018)

Anais do XXIX Simpósio Brasileiro de Informática na Educação (SBIE 2018)

suporte computacional (Computer-supported collaborative learning - CSCL) foi utilizado para a regulação emocional em dois estudos primários (5\%). A teoria de Lazarus, o peer feedback, a recomendação e o treinamento de inoculação de stress foram utilizados em 1 estudo primário (3\%) cada. Vale destacar que somente a teoria de regulação de Gross e Lazarus foram avaliadas experimentalmente na psicologia.

Tabela 1. Distribuição dos estudos por teoria para regulação emocional

\begin{tabular}{|l|c|c|}
\hline Teoria & Frequência & Porcentagem (\%) \\
\hline Gross & 16 & $43 \%$ \\
\hline Não especificado & 15 & $40 \%$ \\
\hline CSCL & 2 & $5 \%$ \\
\hline Lazarus & 1 & $3 \%$ \\
\hline Peer feedback & 1 & $3 \%$ \\
\hline Recomendação & 1 & $3 \%$ \\
\hline Treinamento de inoculação de stress & 1 & $3 \%$ \\
\hline \hline
\end{tabular}

Nas teorias de [Gross 1998] e [Lazarus 1966] são propostas algumas estratégias de regulação emocional (Tabela 2). Na teoria de Gross, o modelo processual da regulação emocional consiste em cinco estratégias onde os indivíduos podem regular suas emoções, sendo elas: seleção da situação, modificação da situação, modificação do foco atencional, mudança cognitiva e modulação da resposta. As estratégias de regulação emocional propostas por [Gross 1998] são as mais utilizadas, correspodendo a 97\% dos estudos primários.

Dentre as estratégias propostas por [Gross 1998], a mudança cognitiva foi a mais utilizada, compondo 13 estudos primários (37\%). A mudança cognitiva consiste na mudança do significado que o indivído atribuiu à situação, por meio da alteração do pensamento sobre a própria situação ou a capacidade de lidar com as exigências da mesma. Uma forma de mudança cognitiva é a Reavaliação Cognitiva [Gross and Thompson 2007], que envolve a modificação do significado atribuído à situação de forma a alterar a resposta emocional a essa situação.

Tabela 2. Distribuição dos estudos por estratégia de regulação emocional

\begin{tabular}{|l|l|c|c|}
\hline Teoria & Estratégia & Frequência & Porcentagem (\%) \\
\hline Gross & Mudança cognitiva & 13 & $37 \%$ \\
\hline & Desenvolvimento da atenção & 6 & $17 \%$ \\
\hline & Indefinido & 4 & $12 \%$ \\
\hline & Modulação da resposta & 4 & $11 \%$ \\
\hline & Seleção da situação & 4 & $11 \%$ \\
\hline & Modificação da situação & 3 & $9 \%$ \\
\hline Lazarus & Coping baseado na emoção & 1 & $3 \%$ \\
\hline \hline
\end{tabular}

A segunda estratégia mais utilizada foi o desenvolvimento da atenção, com 6 estudos primários, correspondendo a 17\%. Essa estratégia permite selecionar qual dos aspectos o indivíduo deseja focar [Gross 1998]. As estratégias de modulação da resposta e seleção da situação (o indivíduo seleciona situações que prefira ou não experienciar) 
VII Congresso Brasileiro de Informática na Educação (CBIE 2018)

Anais do XXIX Simpósio Brasileiro de Informática na Educação (SBIE 2018)

foram encontrados em 4 estudos primários cada. Uma forma de modulação da resposta é a Supressão Emocional, que consiste no esforço do indivíduo em inibir a experiência emocional em curso. A modificação da situação (o indivíduo tenta modificar diretamente a situação) foi a estratégia da teoria de Gross menos utilizada, com 1 estudo primário. 4 estudos primários não definiram quais estratégias da teoria de Gross que foram utilizadas.

Outra teoria para a regulação emocional utilizada foi a coping, proposto por [Lazarus 1966]. O conceito de coping se descreve como o conjunto das estratégias utilizadas pelas pessoas para adaptarem-se as circunstâncias adversas ou estressantes [Folkman and Lazarus 1980]. Essa teoria possui duas estratégias, sendo a coping baseado na emoção (tenta reduzir a intensidade da emoção gerada) e a coping baseado no problema (tenta resolver o problema que gera a emoção).

\section{2. $Q P_{3}$ : Formas de utilizar as estratégias de regulação emocional e $a s \mathbf{Q P}_{4}$ : formas de interação para a regulação}

Nas questões de pesquisa $\mathbf{Q P}_{\mathbf{3}}$ e $\mathbf{Q P}_{\mathbf{4}}$, o objetivo é identificar como a regulação emocional é realizada e quais as formas de interação dentro dos sistemas educacionais, respectivamente.

Foram identificados 8 formas de interação para a regulagem emocional, sendo: realidade virtual (RV), agente pedagógico, algoritmo, fórum/wiki, interface, luz led, jogos sérios e textual. A forma de interação mais utilizada, dentro dos sistemas educacionais com regulação emocional, foi jogos sérios com $21 \%$ (8 estudos primários). Em segundo lugar, pode ser percebido o empate entre as categorias "agente pedagógico" e textual, correspondendo $18 \%$ (7 estudos primários) cada. A categoria algoritmo ficou em terceiro lugar (5 estudos primários, com 13\%), que refere-se a modelos computacionais com regras estatísticas para a regulação emocional. A quarta posição, com 11\% (4 estudos), é ocupada pela categoria interface. Nessa categoria foram classificados sistemas educacionais que utilizam elementos da interface para a regulação emocional, como a modificação de um botão, figuras ou campos de texto. O quinto lugar é ocupado pelas categorias Fórum/Wiki e RV, 8\% (3 estudos primários). E a sexta posição é ocupada pela categoria Luz LED e um moinho de vento, correspondendo a 3\% (1 estudo primário).

A Tabela 3 apresenta de forma resumida todos os estudos primários e como a regulação emocional é realizada para cada forma de interação. Muitos estudos primários não demonstraram como utilizaram as teorias de regulagem escolhidas detalhadamente, como a regulagem realizada por meio da $\mathrm{RV}$, algoritmo, fórum/wiki, interface, luz led e moinho e jogos sérios. Para a lista detalhada, acessar https : / goo.gl / ptGHxW, na aba "Estratégias de Regulação".

\section{3. $\mathrm{QP}_{5}$ : Tipos de ambientes}

A questão de pesquisa $\mathbf{Q} \mathbf{P}_{5}$ tem como objetivo mapear os tipos de ambientes utilizados nos estudos que possuem alguma forma de regulação emocional. Os resultados obtidos foram classificados e agrupados em onze tipos de ambientes.

Com base nos resultados obtidos, o ambiente mais utilizado pelos pesquisadores é o E-learning, com $27 \%$ (11 casos) de representatividade dos trabalhos analisados. O segundo e terceiro lugar são ocupados pelos Jogos educacionais com 17\% (7 casos) e RV (realidade virtual) com 15\% (6 casos), respectivamente. No quarto lugar, observa-se um 
VII Congresso Brasileiro de Informática na Educação (CBIE 2018)

Anais do XXIX Simpósio Brasileiro de Informática na Educação (SBIE 2018)

Tabela 3. Distribuição dos estudos primários por formas de interação e modo de regulação emocional

\begin{tabular}{|c|c|}
\hline Tipo & Regulagem \\
\hline realidade virtual $(\mathrm{RV})$ & $\begin{array}{l}\text { Relaxamento por meio de imersão com fotos de paisagens } \\
\text { e mindfulness }\end{array}$ \\
\hline $\begin{array}{l}\text { Agente } \\
\text { pedagógico (modelo) }\end{array}$ & $\begin{array}{l}\text { Seleção da situação: evitar um estímulo. Desenvolvimento } \\
\text { da atenção: desviar o estímulo. Mudança cognitiva: a } \\
\text { crença é regulada (por exemplo, reavaliação da situação: di- } \\
\text { zer a si mesmo que a situação não é ruim). Mas também } \\
\text { é possível regular o desejo ajustando os objetivos. Supres- } \\
\text { são: supressão de sentimentos experimentados e preparação } \\
\text { / ação (por exemplo, ficar em um local em vez de fugir) }\end{array}$ \\
\hline $\begin{array}{l}\text { Agente } \\
\text { pedagógico }\end{array}$ & $\begin{array}{l}\text { Desenvolvimento da atenção: agente pedagógico propõe } \\
\text { outro material de apoio. Avaliação cognitiva: mostra para } \\
\text { o estudante que mesmo errando, está aprendendo ciência } \\
\text { ou propõe atividades quando o aluno está entediado, como } \\
\text { postar algo no fórum e interagir com os outros }\end{array}$ \\
\hline Algoritmo & $\begin{array}{l}\text { Reavaliação cognitiva por meio da avaliação do "valor" da } \\
\text { tarefa e desenvolvimento da atenção por meio do redirecio- } \\
\text { namento da informação }\end{array}$ \\
\hline Fórum, wiki & $\begin{array}{l}\text { Regulagem por meio do trabalho colaborativo: por meio de } \\
\text { chat, fóruns, área de troca de documentos }\end{array}$ \\
\hline Interface & $\begin{array}{l}\text { Customização realizada pelo próprio usuário quanto ao } \\
\text { acesso das informações/navegação, cores e composição das } \\
\text { informações e regulação por meio de mudança dos elemen- } \\
\text { tos da interface }\end{array}$ \\
\hline $\begin{array}{l}\text { Luz LED e } \\
\text { Moinho de vento }\end{array}$ & $\begin{array}{l}\text { Respirar fundo e fazer algo divertido, tocar uma música } \\
\text { calma, mostrar sua cor favorita, ou apresentar uma comé- } \\
\text { dia automaticamente. }\end{array}$ \\
\hline Jogos sérios & $\begin{array}{l}\text { Modificação do cenário, de música, controles, cores, perso- } \\
\text { nagens, direções, formas ou dificuldade do jogo }\end{array}$ \\
\hline Textual & $\begin{array}{l}\text { Seleção da situação: entrar no seu grupo favorito, conver- } \\
\text { sar com seus bons amigos/professor. Modificação da situ- } \\
\text { ação: trocar os tópicos, conversar com amigos e professo- } \\
\text { res. Modificação cognitiva: recomendar história de encora- } \\
\text { jamento, comunicação de estudante-estudante, comunica- } \\
\text { ção com professor. Desenvolvimento da atenção: ver uma } \\
\text { animação, escutar uma música. }\end{array}$ \\
\hline
\end{tabular}


VII Congresso Brasileiro de Informática na Educação (CBIE 2018)

Anais do XXIX Simpósio Brasileiro de Informática na Educação (SBIE 2018)

empate entre STI (Sistema Tutor Inteligente) e ambientes gerais (por exemplo, modelos que podem ser utilizados em diferentes sistemas) com 10\% (4 casos) de representatividade cada. A quarta posição, com 5\% (2 casos) de representatividade, é ocupada por sistemas educacionais que não foram desenvolvidos para internet e pelo Moodle. Por fim, os ambientes que regulam a emoção do estudante com 3\% (1 caso) de representatividade cada são: chat, MOOC, vídeo educacional e mobile.

\section{4. $\mathrm{QP}_{6}$ : Formas de detecção}

Na questão de pesquisa $\mathbf{Q} \mathbf{P}_{6}$, o objetivo era identificar quais as principais formas de detecção das emoções dos alunos. As formas de detecção identificadas nos trabalhos selecionados foram classificadas nas categorias: linguística, expressões faciais, sinais fisiológicos e dados comportamentais. Cada uma dessas categorias contém subcategorias mais específicas (Tabela 4).

Dentre todas as categorias, pode ser observado que o auto-relato é a forma de detecção mais utilizada dentro dos ambientes educacionais, correspondendo a 35\% (17 estudos primários). Acredita-se que essa forma de detecção seja a mais utilizada por ser a de menor custo e de fácil acesso, entretanto, essa forma não é confiável [Muñoz et al. 2011]. Em segundo lugar, 15\% dos estudos primários não especificaram a forma como detectaram as emoções dos alunos, com 7 casos. Em terceiro lugar, apresenta-se um empate entre capacetes de eletroencefalograma (EEG), log das ações do usuário no sistema e texto, com $6 \%$ (3 estudos primários), cada.

Tabela 4. Categorias por tipo de detecção

\begin{tabular}{|l|l|l|l|}
\hline Categoria & Detecção & Frequência & Porcentagem (\%) \\
\hline- & Não especificado & 7 & $15 \%$ \\
\hline \hline \multirow{3}{*}{ Dados comportamentais } & Log & 3 & $6 \%$ \\
\cline { 2 - 4 } & Observação clínica & 2 & $4 \%$ \\
\hline \hline \multirow{4}{*}{ Expressões faciais } & Face & 3 & $6 \%$ \\
\cline { 2 - 4 } & Eye-tracking & 1 & $2 \%$ \\
\hline \hline \multirow{5}{*}{ Singuística } & Auto-relato & 17 & $35 \%$ \\
\cline { 2 - 4 } & Texto & 3 & $6 \%$ \\
\cline { 2 - 4 } & Voz & 2 & $4 \%$ \\
\cline { 2 - 4 } & Entrevistas & 1 & $2 \%$ \\
\hline \hline \multirow{5}{*}{} & EEG & 3 & $6 \%$ \\
\cline { 2 - 4 } & Eletrocardiograma & 2 & $4 \%$ \\
\cline { 2 - 4 } & Frequência respiratória & 1 & $2 \%$ \\
\cline { 2 - 4 } & Biosensores (Geral) & 1 & $2 \%$ \\
\cline { 2 - 4 } & Galvanic Skin Response & 1 & $2 \%$ \\
\cline { 2 - 4 } & Temperatura corporal & 1 & \\
\hline \hline
\end{tabular}

\section{5. $\mathrm{QP}_{7}$ : Disciplinas em que a regulação emocional têm sido empregadas}

A questão de pesquisa $\mathbf{Q} \mathbf{P}_{7}$ tem como propósito identificar as disciplinas (área de conhecimento) dos ambientes educacionais que possuem regulação emocional. Os resultados obtidos foram classificados e agrupados em 13 disciplinas. 
VII Congresso Brasileiro de Informática na Educação (CBIE 2018)

Anais do XXIX Simpósio Brasileiro de Informática na Educação (SBIE 2018)

Com base nos resultados obtidos, os estudos primários não investigaram a regulação emocional para uma determinada disciplina, ou seja, a proposta de regulação é independente de domínio de conhecimento, sendo encontrados em 18 investigações (correspondendo a 47\%). As categorias "não especificado" (não especificaram as disciplinas), computação e saúde, contaram com 3 estudos primários cada (correspondendo a 8\%). As categorias segurança e biologia ficaram empatadas, com 2 estudos primários cada $(5 \%$ dos estudos). E, finalmente, as disciplinas "lei americana", criatividade, entretenimento, finanças, vendas, "habilidades sociais" e matemática contaram com 1 estudo primário cada, correspondendo a $3 \%$ das investigações.

\section{Ameaças à Validade}

As questões de pesquisa e os critérios de inclusão e exclusão foram criados antes do início do mapeamento, com a finalidade de garantir um processo de seleção imparcial. A seleção dos artigos foi realizada de forma independente entre os autores, respeitando os critérios de inclusão e exclusão definidos previamente. Ao final, eventuais dúvidas e desacordos foram sanados. Conforme descrito na Seção 2, somente um conjunto limitado de base de dados foi utilizado e, portanto, é possível que estudos relevantes não tenham sido incluídos. A coerência do sistema de classificação elaborado pode significar uma ameaça à validade, uma vez que o conhecimento necessário para elaborá-lo muitas vezes é obtido somente ao final da seleção [Pretorius and Budgen 2008].

\section{Conclusão}

Este trabalho teve como objetivo realizar um mapeamento sistemático sobre as pesquisas de regulação emocional em ambientes educacionais com suporte computacional. A partir dos 34 artigos selecionados, foi possível responder às setes questões de pesquisa que guiaram este trabalho, apresentar como as teorias foram utilizadas para a regulação emocional, as formas de interação do sistema com os alunos para que ocorra a regulação, além de buscar identificar como as emoções são detectadas e para quais disciplinas a regulação tem sido investigada.

Foi possível identificar que apesar da maioria dos trabalhos (43\% estudos) utilizarem a teoria de Gross para a regulação emocional, um número significativo deles não aplicou qualquer teoria para a regulação ( $40 \%$ dos estudos primários), respondendo a $\left(\mathbf{Q P}_{1}\right)$. Dentre os estudos que empregam essas teorias, a estratégia emocional mais utilizada é a "mudança cognitiva", com $37 \%$ dos estudos $\left(\mathbf{Q P}_{\mathbf{2}}\right)$. As formas de interação nos sistemas educacionais que auxiliam na regulação emocional foram por meio de jogos sérios $\left(\mathbf{Q P}_{4}\right)$, que utiliza elementos do jogo, como por exemplo, cenários, música, personagens, cores e formas como artifícios para reavaliar uma crença ou auxiliar no foco para regular a emoção $\left(\mathbf{Q P}_{\mathbf{3}}\right)$. O e-learning representa a maior parte dos ambientes onde há regulação emocional $\left(\mathbf{Q P}_{\mathbf{5}}\right)$. Muitos dos trabalhos não especificam como esses sistemas detectaram a emoção para depois agir com a regulação $\left(\mathbf{Q P}_{\mathbf{6}}\right)$. Por fim, os ambientes educacionais investigam a regulação emocional em disciplinas gerais, ou seja, não direcionam a regulação emocional para um determinado assunto $\left(\mathbf{Q} \mathbf{P}_{7}\right)$.

Baseado neste estudo, os autores acreditam que existem alguns direcionamentos às pesquisas de informática na educação para uma contribuição mais relevante na área de regulação emocional em ambientes educacionais. Primeiramente, observa-se que apesar 
VII Congresso Brasileiro de Informática na Educação (CBIE 2018)

Anais do XXIX Simpósio Brasileiro de Informática na Educação (SBIE 2018)

da maioria das pesquisas se basearem em uma teoria psicológica com avaliação experimental para a regulação emocional (e.g. teoria da regulação emocional de Gross), um número significativo ainda não utiliza qualquer teoria. Isso pode prejudicar a confiabilidade dos resultados e a eficácia da regulação emocional. Além disso, as pesquisas que empregam essas teorias, não direcionam as investigações aplicando as estratégias de cada teoria.

Outra questão observada foi identificar em quais disciplinas a regulação emocional é investigada nos ambientes educacionais. A maioria dos estudos encontrados pesquisam a regulação sem o foco em uma determinada disciplina. Entretanto, não é o ideal, pois deve-se levar em consideração o contexto e as diferenças entre os assuntos. Por exemplo, há maior resistência e dificuldade do aluno em aprender álgebra e física, que podem gerar frustração, tédio e confusão. A regulação da emoção enquanto aprendem esses assuntos, podem-se apresentar de modo diferente comparado com assuntos menos evitados, como criatividade ou biologia.

\section{Referências}

Arguedas, M., Daradoumis, T., and Xhafa, F. (2016). Analyzing the effects of emotion management on time and self-management in computer-based learning. Computers in Human Behavior, 63:517-529.

Folkman, S. and Lazarus, R. S. (1980). An analysis of coping in a middle-aged community sample. Journal of health and social behavior, pages 219-239.

Gross, J. J. (1998). Antecedent-and response-focused emotion regulation: divergent consequences for experience, expression, and physiology. Journal of personality and social psychology, 74(1):224.

Gross, J. J. and Thompson, R. A. (2007). Emotion regulation: Conceptual foundations.

Isen, A. M. (2000). Some perspectives on positive affect and self-regulation. Psychological Inquiry, 11(3):184-187.

Lazarus, R. S. (1966). Psychological stress and the coping process.

Malekzadeh, M., Mustafa, M. B., and Lahsasna, A. (2015). A review of emotion regulation in intelligent tutoring systems. Journal of Educational Technology \& Society, 18(4):435.

Muñoz, K., Mc Kevitt, P., Lunney, T., Noguez, J., and Neri, L. (2011). An emotional student model for game-play adaptation. Entertainment Computing, 2(2):133-141.

Oxford, R. L. and Bolaños-Sánchez, D. (2016). A tale of two learners: Discovering mentoring, motivation, emotions, engagement, and perseverance. In New directions in language learning psychology, pages 113-134. Springer.

Pessoa, L. (2008). On the relationship between emotion and cognition. Nature reviews neuroscience, 9(2):148.

Petersen, K., Feldt, R., Mujtaba, S., and Mattsson, M. (2008). Systematic mapping studies in software engineering. In EASE, volume 8, pages 68-77.

Pretorius, R. and Budgen, D. (2008). A mapping study on empirical evidence related to the models and forms used in the uml. In ACM-IEEE ESEM, pages 342-344. 